\title{
The surgical arrest of post-tonsillectomy haemorrhage: Hospital Episode Statistics 12 years on
}

\author{
MS Osborne ${ }^{1}$, MPA Clark² \\ ${ }^{1}$ Queen Elizabeth Hospital Birmingham, University of Birmingham NHS Foundation Trust, \\ Birmingham, UK \\ ${ }^{2}$ Gloucester Royal Hospital, Gloucester, UK
}

\section{ABSTRACT}

INTRODUCTION The risk of returning to theatre for arrest of haemorrhage following tonsillectomy can be determined from analysis of the Hospital Episode Statistics data provided by the Department of Health website. This method was employed previously for data between 1998-2002 and was repeated in this study to observe any changes over this time period.

MATERIALS AND METHODS Hospital Episode Statistics data for England from 2010-2016 were used. The number of tonsillectomies and surgical arrest of post-tonsillectomy haemorrhage were considered for children and adults.

RESULTS Of 267,159 tonsillectomies performed over the six-year period, 5027 (1.88\%) returned to theatre for control of bleeding. This was 3.5 times more likely in adults than children $(P<0.0001)$. Comparison with the previous study showed an increase in return to theatre rates following tonsillectomy of $1.06 \%$, from $0.82 \%$ to $1.88 \%$.

CONCLUSION Adults are more likely than children to require and arrest of haemorrhage post-tonsillectomy. Return to theatre rates have increased since 2004 at an estimated additional cost to NHS England of $£ 1,415,056$ per annum. The causes of this observed increase have yet to be determined.

\section{KEYWORDS}

Tonsillectomy - Consent

Accepted 14 January 2018

\section{CORRESPONDENCE TO}

Max Osborne, E: maxosborne@doctors.org.uk

\section{Introduction}

In 2004, the Hospital Episodes Statistics (HES) related to post-tonsillectomy bleeds between 1998 and2002 were reviewed. ${ }^{1}$ The results demonstrated that there was a $0.82 \%$ rate of return to theatre for control of bleeding following tonsillectomy. It also demonstrated that adults had a higher return to theatre rate $(1.47 \%)$ compared with children $(0.38 \%)$. This study aimed to repeat the analysis with HES data between 2010 and 2016.

The Department of Health provides HES, which is a dataset containing records of all patients admitted to NHS hospitals in England and what procedures have been performed. ${ }^{2}$ Private hospitals are not included, although private patients treated in NHS hospitals are. The database does not give specific individual information on patient admissions but does give an overview of current surgical rates in England. The data are available on 'main procedures and interventions', which can be subdivided into four-character OPCS-4 codes and the statistics related to them. The HES data are limited by data entry errors as they rely on the correct coding of all operative procedures.

\section{Materials and methods}

'Main procedures and interventions' data for the available years (2010-2016) were downloaded in Microsoft Excel format. The operative (OPCS-4) codes F34.1, F34.2, F34.3, F34.4, F34.8 and F34.9 relating to tonsillectomy procedures and code F36.5 relating to the surgical arrest of post-tonsillectomy bleeding were considered. Codes regarding excision on tonsil remnant (F34.5), excision of lingual tonsil (F34.6) and coblation tonsillectomy (F34.7) were excluded from the study. Data for children ( $0-14$ years) and adults (15-75+ years) were compared and statistically assessed with a chi-square test. This was the identical methodology used in the previous audit, where the HES data were only available in these two age categories. ${ }^{1}$ Recent HES data are provided in 24 age categories, which were amalgamated to allow for useful comparison.

\section{Results}

Between April 2010 and April 2016, 267,159 tonsillectomies were performed, 5027 (1.88\%) of which returned to theatre 


\begin{tabular}{|c|c|c|c|c|c|c|c|c|c|}
\hline \multirow[t]{2}{*}{ Years } & \multicolumn{3}{|c|}{ Tonsillectomy ( $n$ ) } & \multicolumn{3}{|c|}{$\begin{array}{l}\text { Surgical arrest of post-tonsillectomy } \\
\text { haemorrhage }(n)\end{array}$} & \multicolumn{3}{|c|}{ Return to theatre (\%) } \\
\hline & $0-14$ years & $15-75+$ years & Total & $0-14$ years & $15-75+$ years & Total & $0-14$ years & $15-75+$ years & Total \\
\hline 2010-11 & 24,708 & 19,009 & 43,717 & 162 & 440 & 602 & 0.66 & 2.31 & 1.39 \\
\hline 2011-12 & 24,243 & 18,483 & 42,726 & 192 & 505 & 697 & 0.79 & 2.73 & 1.63 \\
\hline $2012-13$ & 24,650 & 19,421 & 44,071 & 181 & 638 & 819 & 0.69 & 3.29 & 1.85 \\
\hline 2013-14 & 26,229 & 20,415 & 46,644 & 282 & 687 & 960 & 1.08 & 3.37 & 2.06 \\
\hline 2014-15 & 26,264 & 19,873 & 46,137 & 300 & 719 & 1019 & 1.14 & 3.62 & 2.21 \\
\hline 2015-16 & 24,806 & 19,058 & 43,864 & 1375 & 3661 & 5027 & 1.04 & 3.53 & 2.12 \\
\hline Totals & 150,900 & 116,259 & 267,159 & 1375 & 3661 & 5027 & 0.91 & 3.15 & 1.88 \\
\hline Previous study ${ }^{1}$ & 131,577 & 88,920 & 220,497 & 500 & 1304 & 1804 & 0.38 & 1.47 & 0.82 \\
\hline
\end{tabular}

for control of postoperative bleeding. In children, 150,900 tonsillectomies were performed and $1375(0.91 \%)$ returned to theatre. In adults, 116,259 were performed and 3661 $(3.15 \%)$ returned (Table 1). Adults were, therefore, 3.5 times more likely than children to return to theatre for control of bleeding.

The difference between the incidence of adults and children returning to theatre was statistically significant (chi square $1707 ; P<0.0001)$. This result is comparable with that of the previous audit (chi-square $771 ; P<0.0001$ ), further supporting this to be a real trend. Although the average number of tonsillectomies has fallen by $19.3 \%$ since 2004 (Table 2), comparisons made with the previous study

Table 2 Total tonsillectomies performed during each year of this and previous comparison studies.

\begin{tabular}{ll} 
Year & Tonsillectomies $(n)$ \\
Clark and Waddell (2004): ${ }^{1}$ & \\
1998-99 & 67,139 \\
1999-2000 & 59,117 \\
2000-01 & 46,001 \\
2001-02 & 48,321 \\
Average & 55,145 \\
This study: & \\
2010/11 & 43,717 \\
2011/12 & 42,726 \\
2012/13 & 44,071 \\
2013/14 & 46,644 \\
2014/15 & 46,137 \\
2015/16 & 43,864 \\
Average & 44,527 \\
\hline
\end{tabular}

demonstrated an overall increase in return to theatre rates from $0.82 \%$ to $1.88 \%$. The pattern is replicated in children $(0.38 \%$ to $0.91 \%)$ and in adult $(1.47 \%$ to $3.15 \%)$ subgroups.

The data analysis also demonstrated an increase in return to theatre rate year on year from $2010(1.39 \%)$ to 2015 (2.21\%; Fig 1$)$. The $1.06 \%$ increase results in an additional 472 emergency procedures per year, based on the average number of tonsillectomies $(44,527)$ performed per year included in this study period (Table 2). The cost of these procedures to NHS England is conservatively estimated at $£ 1,415,056$ (HRG, CA81B, non-elective tariff). ${ }^{3}$

\section{Conclusion}

This study shows that adults are 3.5 times more likely than children to require a return to theatre for arrest of haemorrhage following tonsillectomy. The overall return to theatre rates have increased by $1.06 \%$ since 2004 . In both adults and children, this rate has more than doubled since the previous audit. These new figures should be taken into account when consenting patients prior to tonsillectomy.

The more challenging question is to consider why this trend has occurred. In early 2010, the Scottish Intercollegiate Guideline Network guidelines were published. ${ }^{4}$ With this came the introduction in the UK of stricter criteria to be applied to obtain funding for tonsillectomy. ${ }^{5}$ Patient selection has become more controlled, which may result in tonsillectomy being only performed on those patients who have experienced more episodes of tonsillitis compared with the first audit. One could argue that this in turn could lead to a more scarred tonsil bed and a more challenging dissection, thereby increasing the risk of postoperative bleeding.

The decline in the overall number of tonsillectomies performed may also have led to less surgical experience for middle-grade surgeons in this procedure, which might in part reflect the observed higher return to theatre rate or indeed, as a result of reduced experience, the junior staff may have a lower threshold for returning to theatre to control a postoperative haemorrhage. 
Percentage of tonsillectomies performed returning to theatre 2010-2016

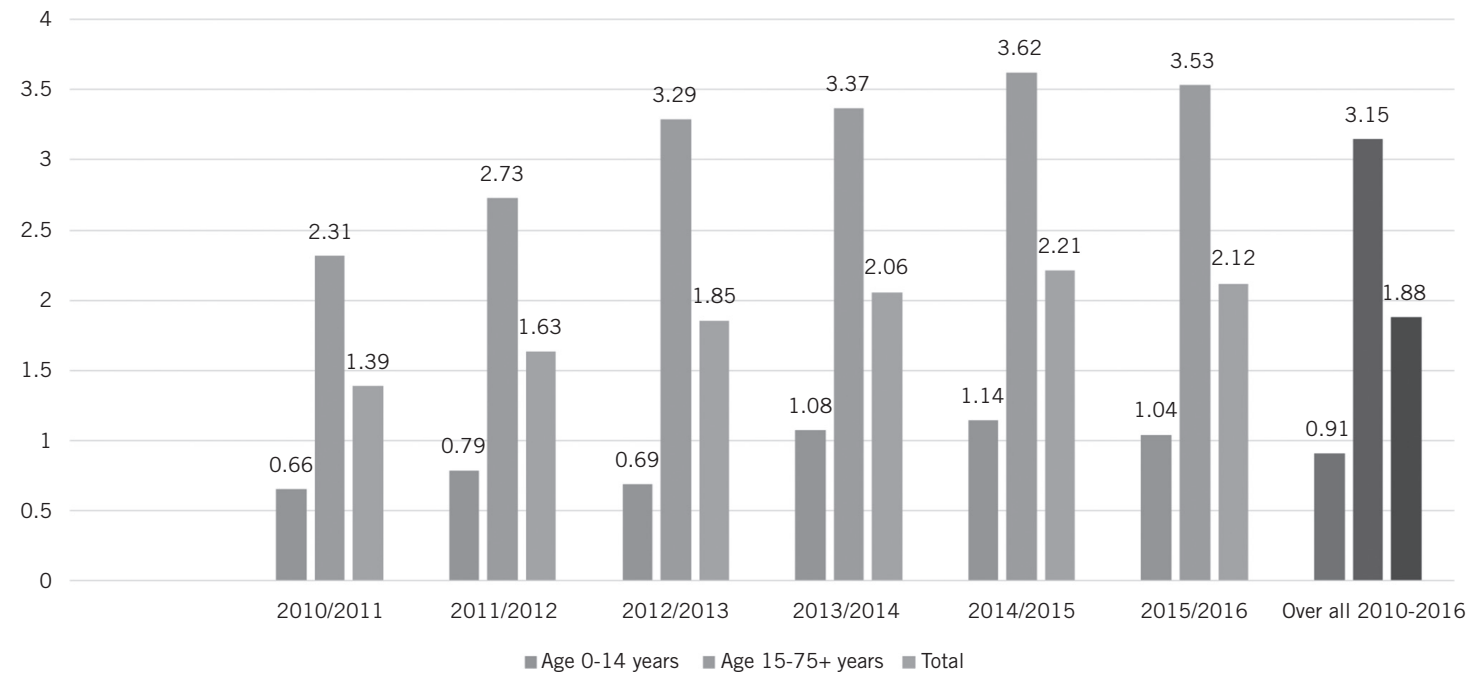

Figure 1 Percentage of tonsillectomies performed returning to theatre from 2010 to 2016

It is important to note that HES data are based upon the correct clinical coding of procedures and interventions; improvements in data reporting and collection methods may partially explain the increase as compared with 2004. This, however, is unlikely to fully explain the year on year increase in rates from 2010 to 2015 shown in the study. Further investigation should be undertaken to ascertain why rates of return to theatre are continuing to increase.

\section{References}

1. Clark MPA, Waddell A. The surgical arrest of post-tonsillectomy haemorrhage: Hospital Episode Statistics. Ann R Coll Surg Engl 2004; 86: 411-412.
2. NHS Digital. Hospital Episode Statistics. https://digital.nhs.uk/search?q=hospital +episode+statistics\&s=s (cited January 2018).

3. NHS Improvement. National tariff payment system $2017 / 18$ and $2018 / 19$. https://improvement.nhs.uk/resources/national-tariff-1719/\#h2-annexes (cited January 2018).

4. Scottish Intercollegiate Guideline Network. Management of Sore Throat and Indications for Tonsillectomy: A national clinical guideline. No. 117. Edinburgh: SIGN; 2010.

5. NHS England. Interim Clinical Commissioning Policy: Tonsillectomy. Ref: N-SC/ 033; November 2013. https://www.england.nhs.uk/commissioning/wp-content/ uploads/sites/12/2013/11/N-SC033.pdf (cited January 2018). 